\title{
The effect of blood composition on T1 mapping
}

Stefania Rosmini MD, $\mathrm{PhD}^{\mathrm{a}}$, Heerajnarain Bulluck MBBS, $\mathrm{PhD}^{\mathrm{b}}$, Amna Abdel-Gadir $\mathrm{MBBS}^{\mathrm{a}}$, Thomas A Treibel MBBS, PhD ${ }^{\mathrm{a}}$, Veronica Culotta MDa, Richard Thompson $\mathrm{PhD}^{\mathrm{c}}$, Stefan K Piechnik PHD, MSCEE ${ }^{\mathrm{d}}$, Peter Kellman $\mathrm{PhD}^{\mathrm{e}}$, Charlotte Manisty $\mathrm{PhD}^{\mathrm{a}}$, James C Moon MD ${ }^{\mathrm{a}, \mathrm{b}}$

${ }^{\text {a }}$ Barts Heart Centre, St Bartholomew's Hospital, London, UK.

${ }^{\mathrm{b}}$ Institute of Cardiovascular Science, University College London, UK

c Department of Biomedical Engineering, Faculty of Medicine and Dentistry, University of Alberta, Edmonton, Canada.

${ }^{\mathrm{d}}$ Division of Cardiovascular Medicine, Radcliffe Department of Medicine, University of Oxford, Oxford, UK.

e National Heart, Lung and Blood Institute, National Institutes of Health, Bethesda, USA.

Word count: 712

Emails:

SR: $\quad$ sterosmini@gmail.com, HB: h.bulluck@gmail.com>, $\quad$ TAT: thomas.treibel@gmail.com, AAG: amnagadir@yahoo.co.uk, VC: veroculotta@gmail.com, RT: richard.thompson@ualberta.ca, $\quad$ SP: stefanp@fmrib.ox.ac.uk, PK: kellmanp@nhlbi.nih.gov, CM: cmanisty@gmail.com, JCM: j.moon@ucl.ac.uk

This research was conducted at The Heart Hospital, 16-18 Westmoreland Street, London, W1G 9PH, UK (has now merged with Barts Heart Centre).

Conflicts of interest: none

Funding: This work was supported by Borse di studio SIC e MSD Italia-Merck Sharp \& Dohme, the Rosetrees Trust, GlaxoSmithKline, and the National Institute for Health Research (NIHR) Oxford Biomedical Research.

\section{Corresponding author:}

Professor James Moon

Barts Heart Centre

St. Bartholomew's Hospital,

West Smithfield, London EC1A 7BE

T: +44 2034656336

F: +440234563086

Email: j.moon@ucl.ac.uk 
Native myocardial T1 and extra-cellular volume (ECV) by cardiovascular magnetic resonance (CMR) are key biomarkers for assessing several heart muscle diseases. Their measurement is however influenced by blood T1, either due to myocardial vascular blood volume or via blood pool partial voluming. Different factors including Hematocrit (Hct), pH, temperature, gender, and oxygen pressure are known to affect blood T1 $(1,2)$ but the influence of blood biological constitutents such as iron, lipids or albumin is unknown.

We sought to assess this. With appropriate ethical approval and written informed consent, we prospectively recruited seventy-seven healthy volunteers free of cardiovascular disease or diabetes, with normal 12-lead ECG and normal CMR. T1 mapping was performed at $1.5 \mathrm{~T}$ using all three primary approaches to the technique: MOdified Look-Locker Inversion Recovery (MOLLI) (3), Shortened Modified LookLocker Inversion recovery (ShMOLLI) (4) and SAturation recovery Single-sHot Acquisition (SASHA) (5). Just prior to the scan, a blood sample was drawn and hemoglobin $(\mathrm{Hb})$, Hct, N-terminal pro b-type natriuretic peptide (NT-proBNP), creatinine, estimated Glomerular Filtration Rate (eGFR), iron, total iron binding capacity (TIBC), ferritin, albumin, HbA1c, non-fasting lipid profile including triglycerides (TG), total cholesterol, low density lipoprotein (LDL) and high density lipoprotein (HDL) cholesterol were tested. T1 values were measured in blood and myocardium before and after administration of extracellular MR contrast medium (3). Univariable and multivariable linear regression analyses were performed to identify biochemical factors affecting blood $\mathrm{T} 1$. 
The population mean age was $49 \pm 14$ years old, range $20-76.38 / 77$ (49\%) were male (mean age: males $50 \pm 14$, females $49 \pm 15$ years old). For MOLLI, ShMOLLI and SASHA respectively, myocardial T1s were $1027 \pm 38,959 \pm 31$ and $1144 \pm 48 \mathrm{~ms}$; and blood T1s were $1638 \pm 78 \mathrm{~ms}, 1543 \pm 77 \mathrm{~ms}, 1587 \pm 103 \mathrm{~ms}$. As expected, as Hct rose, blood T1 fell for all sequences (ShMOLLI $\left.r^{2} 0.530, p<0.0001\right)$, MOLLI $\left(r^{2} 0.452\right.$, $\mathrm{p}<0.0001)$, and SASHA $\left(\mathrm{r}^{2} 0.375, \mathrm{p}<0.0001\right)$.

However, univariable analysis (here ShMOLLI - other sequence concordant) showed additional correlations with iron bound to transferrin $\left(r^{2}=0.405, p<0.0001\right)$, HDLcholesterol $\left(r^{2}=0.229, p<0.0001\right)$, creatinine $\left(r^{2}=0.115, p<0.002\right)$, ferritin $\left(r^{2}=0.101\right.$, $\mathrm{p}=0.003)$, TG $\left(\mathrm{r}^{2}=0.097, \mathrm{p}=0.003\right)$ and LDL-cholesterol $\left(\mathrm{r}^{2}=0.031, \mathrm{p}=0.069\right)$. Albumin, total cholesterol, NT-proBNP, eGFR and HbA1c were not associated with blood T1 (by any sequences, log transformed where needed).

Multivariable analysis showed that blood T1 is largely determined by just three variables, in descending order of effect: Hct, iron and HDL-cholesterol regardless of sequences (MOLLI: cumulative $\mathrm{r}^{2}=0.778$, ShMOLLI: $\mathrm{r}^{2}=0.772$, SASHA: $\mathrm{r}^{2}=0.567$, all $\mathrm{p}<0.0001$ ), with SASHA performance slightly lower than MOLLI and ShMOLLI.

Measured blood T1 is associated with measured myocardial T1 (correlation $\mathrm{r}^{2}=0.266$, $\mathrm{p}=0.01$ by MOLLI; $\mathrm{r}^{2}=0.374, \mathrm{p}=0.001$ by ShMOLLI and $\mathrm{r}^{2}=0.578, \mathrm{p}<0.0001$ by SASHA). ECV is similarly associated by blood $\mathrm{T} 1\left(\mathrm{r}^{2}=0.392, \mathrm{p}=0.001\right.$ by MOLLI; $r^{2}=0.464$ by ShMOLLI and $r^{2}=0.457$ by SASHA with $p<0.0001$ respectively).

To summarise, blood myocardial T1 is primarily determined by blood biology with three parameters, Hct, iron and HDL-cholesterol, out of the ones here investigated, explaining almost $80 \%$ measured variability in health. Anemia, iron deficiency and a high HDL-cholesterol, all more common in women, increase blood T1 and appear to explain this typical female longer blood T1. Native blood T1 is associated with 
myocardial T1 and ECV. This may be from the inclusion of blood signal in measured myocardial T1, but also potentially from biological processes (e.g. compensatory vasodilatation in anemia) - further work is required to understand this better.

Figure - Correlation between ShMOLLI blood T1 and Hct, iron, HDLcholesterol and albumin.

Caption: Figure 1 shows significant correlations between blood T1 and Hct, blood T1 and iron and blood T1 and HDL-cholesterol while correlation between blood T1 and albumin was not significant.
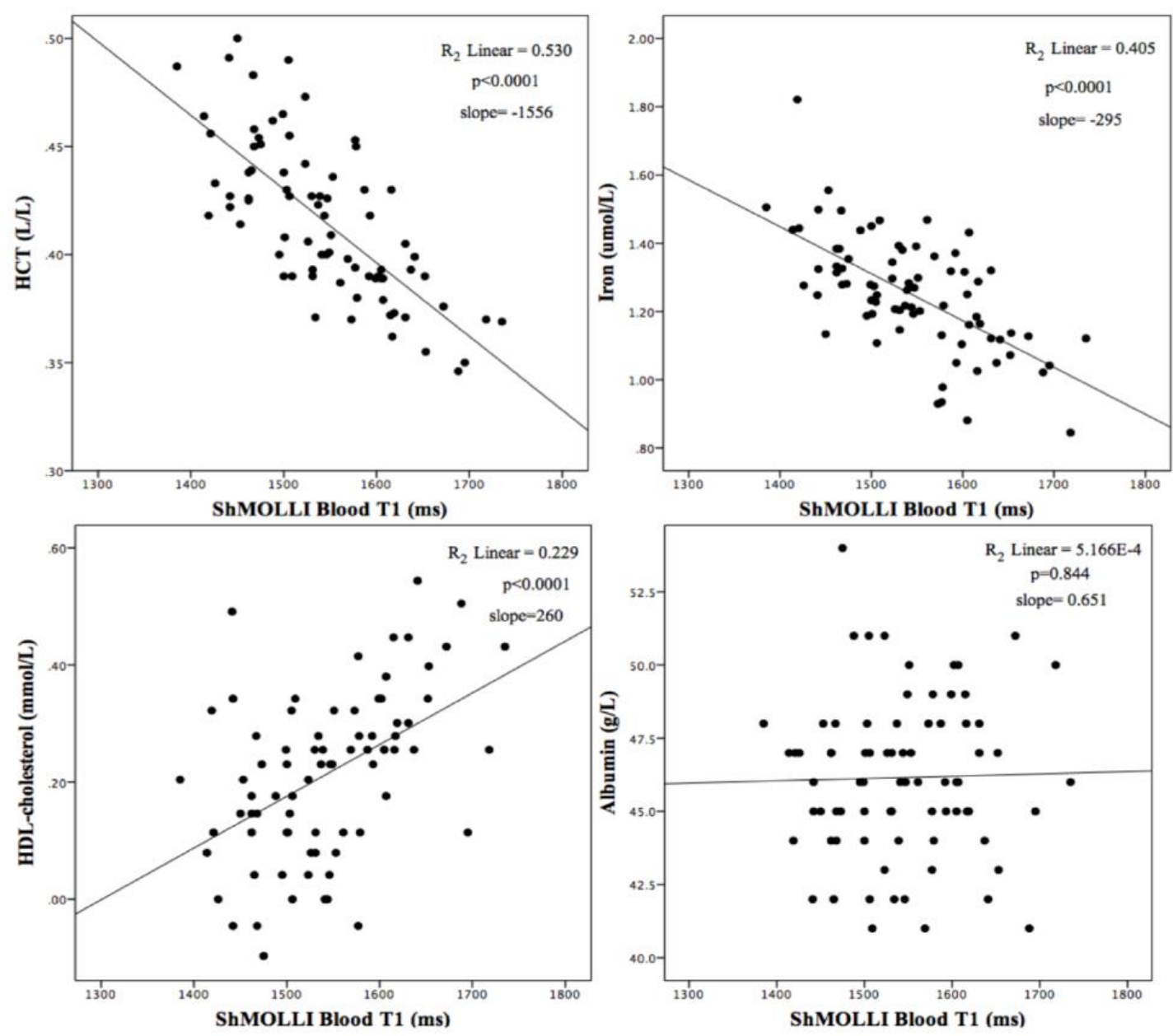

\section{References}


1. Silvennoinen MJ, Kettunen MI, Kauppinen RA. Effects of hematocrit and oxygen saturation level on blood spin-lattice relaxation. Magn Reson Med. 2003;49:568-71.

2. Lu H, Clingman C, Golay X, van Zijl PC. Determining the longitudinal relaxation time (T1) of blood at 3.0 Tesla. Magn Reson Med. 2004;52:679-82.

3. Kellman P, Hansen MS. T1-mapping in the heart: accuracy and precision. J Cardiovasc Magn Reson. 2014;16:2.

4. Piechnik SK, Ferreira VM, Dall'Armellina E, et al. Shortened Modified LookLocker Inversion recovery (ShMOLLI) for clinical myocardial T1-mapping at 1.5 and $3 \mathrm{~T}$ within a 9 heartbeat breathhold. J Cardiovasc Magn Reson. 2010;12:69.

5. Chow K, Flewitt JA, Green JD, et al. Saturation recovery single-shot acquisition (SASHA) for myocardial T(1) mapping. Magn Reson Med. 2014;7 
